\title{
LA PRESCRIPCIÓN DE LOS DELITOS EN EL DERECHO ROMANO ${ }^{54}$
}

\author{
J.A.C. THOMAS \\ (Glasgow)
}

El punto de partida de cualquier consideración sobre este tema debe ser:

C. 9.99.12 (Impp. Diocletianus et Maximianus AA et CC. Primo) "Querela falsi temporalibus praescriptionibus non excluditur nisi viginti annorum excepetione, sicut cetera quoque fere crimina » (A.D. 293)

Este decreto de Diocleciano y Maximiano es aceptado por los tratados clásicos de Mommsen ${ }^{55}$ y Ferrini ${ }^{56}$ como muestra de que en tiempos de Diocleciano existió un término general de veinte años para la prescripción de la responsabilidad criminal, en ausencia de cualquier otra disposición específica. Hace unos treinta años, aproximadamente, el Profesor Volterra - en lo que continúa siendo el único estudio específico sobre el tema ${ }^{57}$ - rechazó la validez de este punto de vista y mantuvo que, por el contrario, el principio general durante todo el siglo III (e incluso antes) era que la responsabilidad criminal no estaba sujeta a un límite temporal. En los últimos años ha sido seguido en lo esencial por el Profesor Amelotti en su estudio general de la Prescripción ${ }^{58}$. Mi modesta opinión, objeto de este bre-

54 Título original: "Prescription of Crimes in Roman Law», en Revue internationale des droits de l'antiquité, IX, 1962, págs. 27 y ss. Traducido por Félix María Pedreira González.

55 Römisches Strafrecht, 487 ss.

56 Diritto Penale Romano (1899), 341 ss.

5737 B.I.D.R. (1929), 57 ss.

58 La Prescrizione delle Azioni in Diritto Romano (1958), 160 ss. 
ve escrito, es sugerir que los argumentos contra la postura tradicional no son de hecho tan sólidos como cabría suponer.

Desde el principio, debe reconocerse que nuestra autoridad sobre los textos en cualquier sentido es extremadamente insuficiente y que muchos de los argumentos del pasado lo han sido por deducción. El núcleo de la controversia, sin embargo, es el «nisi ... exceptione» de la C. 9. 22.12. Esto es mantenido con cierta debilidad por Volterra ${ }^{59}$; Amelotti va más allá y suprimiría todo el pasaje después de «excluditur» $^{60}$. Y mientras Ferrini ${ }^{61}$ sugiere un origen post-Antonino pero pre-Diocleciano para el periodo de veinte años (para usar el término apropiado de Scot) ${ }^{62}$, Volterra y Amelotti mantuvieron que nos encontramos ante una innovación de Justiniano ${ }^{63}$.

Un aspecto preliminar adicional: es posible la existencia de un plazo general de prescripción en el siglo tercero, lo que es el núcleo de la discusión. Podemos obviar desde ahora el hecho conocido y no discutido de que algunos delitos (generalmente por sus estatutos de creación) respondían a específicos y más cortos plazos de prescripción — "adulterium» y sus delitos asociados ${ }^{64}$, "peculatus» ${ }^{65}$, y la apertura del testamento de otro...6 ${ }^{66}$, por tomar los ejemplos obvios-, mientras que otros eran tan atroces como para posibilitar su persecución a lo largo de toda la vida (y, en algunos casos, después de la muerte $^{67}$ ) del malhechor — "maiestas» $\mathrm{o}$ "perduellio» ${ }^{68}$, "parricidium" ${ }^{69}$ (en su sentido técnico), «accusatio suppositi partus» ${ }^{70}$ y el tardío delito de apostasía ${ }^{71}$ - Estos, por supuesto, no conllevan dificultad. Lo que

\footnotetext{
${ }^{59}$ Op. cit. p. 69.

60 Op. cit. p. 165.

61 Op. cit. p. 342 y n.
}

${ }^{62}$ Quizás pueda ser de interés que la cuestión de un periodo de prescripción de veinte años en materia criminal en Roma fue discutida por los casos escoceses, MACGREgOR (1773) M. 11, 146, y más recientemente, SugdEN (1934) J.C. 103.

63 VolTERRA, op. cit.; AMELOTTI, cit., pp. 165/7.

${ }^{64}$ Cf. 48.54.1, 12.4/6, 15.2; 48.16.1.10, 12.6, 16, 30.5. Ver, en general, AMELoTTI, cit., 165, n. 168 para referencias adicionales.

6548.13 .9

66 29.5.13; cf. VolterRa, cit., p. 63, FERRINI, cit., 343; y ver después p. 430 n. 66.

67 En los requisitos y evolución del presente documento, ver VOLTERRA, 3 RIDA (= 2 Mél de Visscher) 485 ss.

68 E.g., C. 1.5.4.4.

69 E.g., 48.9.10 (comparar homicidio común - e.g., C. 9.6.3).

${ }^{70}$ Ver 48.10.19.1. Las especiales disposiciones de la acusación muestran que esto ha sido seriamente tenido en cuenta: por tanto no parece necesario denegar la posible permanencia de la «accusatio suppositi partus ...», FERRINI, cit. 343 - pero, por supuesto, ello no invalida la perspectiva de su interpretación.

71 C. 1.7 .4 y cf. C.Th. 9.38.6. 
nos preocupa es el delito común, si puede decirse así, sin periodo de tiempo determinado. Además, todos los autores reconocen que no existía un periodo general de limitación en la República o en los primeros siglos del Imperio ${ }^{72}$. Es precisamente la C. 9.22.12 la que primero manifiesta ese límite de veinte años y la que constituye, de hecho, nuestra única prueba de ello. De aquí la controversia sobre su falta de autenticidad; hay tantos puntos en común, que el ataque contra la C. 9.22.12 es realmente importante. Los que niegan que proceda de Diocleciano y se la adjudican a Justiniano deben admitir que, en tiempos de Justiniano, debieron ser leídas disposiciones aparentemente en conflicto - conflicto en el sentido de que no indicaban la existencia de un límite temporal (textos que deberemos tener en cuenta a su debido tiempo ${ }^{73}$ ) - en el contexto de la compilación, como tácitamente sujetas al periodo de veinte años ${ }^{74}$.

Entonces cualquiera podría preguntarse, ¿por qué no fue posible antes esta tácita limitación? Esto es, ¿por qué no pudo la inferencia de un límite general de veinte años, en ausencia de otra específica previsión, retroceder al siglo tercero, de modo que esos otros textos de Constantino, etc., estuvieran ya sometidos al plazo en el momento de su declaración?

Pero, primero, echemos un vistazo a los argumentos formales. El Profesor Volterra pone reparos al «nisi ... exceptione» como una inserción típica, con su característica introducción "nisi», y al uso de la palabra "exceptio» para indicar la limitación temporal. Habiendo observado que «exceptio» era un término técnico del procedimiento formulario, advierte que el otro único uso de "exceptio" para "prescriptio» se encuentra en la C. 9.9.27.(28), lo que señala que está manifiestamente interpolado ${ }^{75}$. Por desgracia, no he podido ver el trabajo en el que el Profesor Volterra atacaba esta disposición imperial ${ }^{76}$; en mi opinión, con todo el respeto, suspendería el juicio de interpolación en ese contexto. Creo que es de interés que la C. 9.9.27(28) sea también de Diocleciano y referida a un tema penal ("adulterium»), con una yuxtaposición de los términos "praescriptio» $\mathrm{y}$ "exceptio» similar a la de nuestro principal objeto de consideración:

\footnotetext{
${ }_{72}$ MOMmSEn, cit. 487, FERRINI, cit., 341, VolTERRA, BIDR cit., 64, AMELOTTI, cit. 160.

${ }^{73}$ Post, pp. 425 ss.

74 Cf. VOLTERRA, BIDR cit., p. 74.

75 BIDR cit., 69-70.

${ }^{76}$ Nota critica alla L. 28(29) ad leg. Iul. de Ad. 9,9 (Spoleto, 1929)
} 
C. 9.9.27(28) (Impp. Diocletianus et Maximianus AA. Concordio) "Ita nobis pudor cordi est, ut removeamus prisci iuris ambages et constituamus in adulterii quaestione abolitis de medio ceteris praescriptionibus praeter quinquenii temporis et lenocinii quod marito obicitur exceptionem, illam etiam, quae post solutum prius matrimonium ante denuntiationem nuptae competit, fidem criminis nosci, indignum est enim, ut ultionem pudoris praestigiae versuti iuris excludant ". (A.D. 295).

También hay que tener en cuenta, como observa Amelotti ${ }^{77}$, que hay menos sutileza en las expresiones "exceptio» $\mathrm{y}$ "praescriptio» en el periodo de la «cognitio». Entonces se descubre, en los textos clásicos, que se habla de límites temporales desde el punto de vista del acusado; tomemos dos ejemplos:

48.5.16(15).7 (Ulpianus, lib. ii de adult.) " Praescriptiones, quae obici solent accusantibus adulterii, ante solent tractari, quam quis inter reos recipiatur: ceterum posteaquam semel receptus est, non potest praescriptionem obicere».

C. 9.9.5 (Imp. Alexander A. Vandati) « Adulter post quinquennium quam commissum adulterium dicitur, quod continuum numeratur, accusari non potest: easque praescriptiones legibus reis datas auferri non oportet ». (A.D. 223).

Nuevamente se puede observar - también desde Dioclecianola C. 9.22.20, donde la construcción «non nisi dolo» tiene el mismo propósito que el «non excluditur» incluido en el, según se dice, pasaje controvertido de la C. 9.22.12:

C. 9.22 .20 (Impp. Diocletianus et Maximianus AA. Et CC. Rufino) « Nec exemplum precum editionis aliter per errorem scriptum cum non nisi dolo falsum committentes crimini subiugentur, cognitionem dati iudicis moratur " (A.D. 294).

Se puede por supuesto intentar arrojar dudas sobre todas estas cosas. Sugeriría que la combinación de circunstancias nos posiciona a favor del carácter no genuino de todo.

Queda además un aspecto: si uno acepta la supresión del profesor Volterra, entonces, como observa Amelotti ${ }^{78}$, la construcción resultante sería formalmente extraña: un decreto que afirmaba que la "querela falsi» no está sujeta a una limitación temporal, sería improbable que añadiese gratuitamente "como muchos otros delitos". Esta es la razón por la que Amelotti también suprimiría el pasaje final.

${ }^{77}$ Op. cit., p. 16 y p. 169.

78 Op. cit., p. 165. 
Pero eso también parece insatisfactorio. Si la referencia a la limitación de veinte años fue debida a Justiniano - a quien se atribuye el haber introducido la limitación - ¿habría tenido que añadir la última frase?

Si, no obstante, como yo creo, la forma poco elegante del «non... nisi... exceptione» no es un obstáculo insuperable, se presenta una solución más satisfactoria. La queja de Amelotti sobre el «sicut...», es en esencia válida: no es probable que un decreto haya hecho una referencia tan general, en lo que tiene todos los signos de ser un apéndice. Pero, si el «non... nisi... exceptione» de la C. 9.22.12 es válido, la locución «sicut» bien podría ser el comentario de algún erudito postclásico, que, como otras muchas glosas y comentarios, se ha introducido en el texto en el curso de la transmisión. En resumen, de lo que hay que sospechar no es de la referencia al periodo de veinte años, sino del «sicut...».

Existe además el argumento de que, si Justiniano fuese el artífice del plazo de prescripción de veinte años, es mucho más probable que hubiese incluido en el Código su propia constitución, imponiéndolo, y entonces se habría referido a ello y, donde hubiese sido apropiado, habría modificado, de acuerdo con ello, otros textos. Véase, por ejemplo, la C. 9.44.3 y compárese la C. 9.44.1 y 2, con la C. Th. 9.36.1 y 2, y véase también la C. 3.1.13: estos textos son relevantes, porque se refieren a la duración del proceso penal una vez comenzado, un campo en el que Justiniano también intervino. Cuando uno recuerda la forma en la que él relata su propia solución a la «longi temporis praescriptio" civil, es muy difícil creer que, si la limitación penal de veinte años fue obra de Justiniano, la hubiésemos encontrado sólo introducida, sin más, como por una casualidad ${ }^{79}$. Todo lo que propongo va a sugerir la validez de la referencia a los 20 años en la C. 9.22.12.

II

Ahora, si uno está preparado para aceptar la validez formal del importante fragmento de la C. 9.22.12, surgen unas dificultades fundamentales. ¿No es sorprendente que no tengamos otra indicación de la introducción del periodo de limitación? Y, en cualquier caso, ¿de dónde proviene?

${ }^{79}$ Cf., la dificultad de Amelotti, p. 165/7 
La conexión preferida, en la teoría tradicional, se lleva a cabo con la limitación de los procedimientos fiscales ${ }^{80}$. El Profesor Volterra pone de manifiesto las disposiciones fiscales, pero sin relacionarlas con el periodo penal de veinte años ${ }^{81}$; Amelotti, por así decirlo, rechazó sin miramientos la posibilidad de tal asociación, como también cualquier conexión con el periodo de 20 años de "longi temporis praescriptio" ${ }^{82}$.

Para ocuparse primero de los derechos fiscales — ciertamente una materia de interés para el Imperio- nos fue dicho por Calistrato que Tito (¿Antonino Pío?) introdujo el periodo de 20 años en conexión con un particular aspecto sobre las propiedades de los fugitivos de la justicia:

49.14.1.3 (Callistratus, lib. prim de iure fisc.) « Preaescriptio autem viginti annorum, quae etiam circa requirendorum adnotatorum bona observatur, ex constitutione divi Titi solet ex eo numerari, ex quo quid ad fiscum pertinere potuit ».

Pero la generalización de la limitación de los veinte años, en los casos en los quie no había otra limitación temporal específica -y había variedad de periodos de prescripción, por ejemplo, para defraudación de renta ${ }^{83} \mathrm{y}$ "nunciatio of bona vacantia» ${ }^{84}$-, fue obra de Severo y Caracalla, como nos fue contado por Marciano:

48.17.3 (Marcianus, lib. sec. de publ. iud) « quamcumque enim quaestionem apud fiscum, si non alia sit propria praescriptio, viginti annorum silentio praescribi divi principes voluerunt ${ }^{85}$.

Ferrini sugiere que la limitación de veinte años de los procedimientos fiscales fue la causa de la limitación temporal similar para el delito, pero sin indicación de el por qué ${ }^{86}$.

Me aventuraría a sugerir que hay factores que lo hacen probable y que también, hasta cierto punto, hacen relevante la referencia a la «longi temporis praescriptio», a pesar de la previa unanimidad sobre la irrelevancia de esta posterior institución ${ }^{87}$.

La «longi temporis praescriptio», puede reconocerse de buen grado, estaba reservada a las acciones civiles; y los derechos fiscales podían

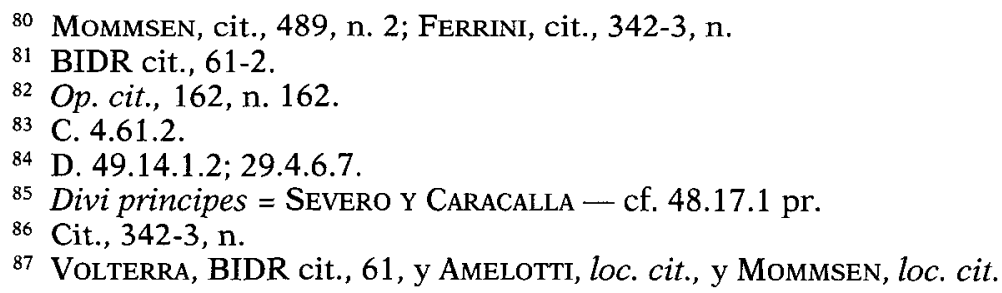


ser considerados como una especial categoría. Pero, naturalmente, los temas fiscales son una materia de interés para el Imperio; y la "longi temporis praescriptio" pertenece esencialmente al procedimiento de la "cognitio» ${ }^{88}$ e, inicialmente, como en general es reconocido $^{89}$, aplicada especialmente respecto a las provincias, es decir, de nuevo una preocupación fundamental del Imperio ${ }^{90}$. Durante el Principado tardío, fue ya incrementándose el uso del procedimiento «cognitio» - esto naturalmente continuó bajo Diocleciano y condujo, ya lo creo, a la abolición oficial de la "formula», como en el 342 ${ }^{91}$. Es claro que por el tercer siglo, también en materia penal, la jurisdicción fue efectivamente ejercida por funcionarios imperiales ${ }^{92}$. En resumen, el procedimiento "cognitio» fue común en ambos procedimientos civil y penal.

Por decirlo así, la discusión previa se ha preocupado en exceso de la distinción entre los derechos civiles y la responsabilidad crimi$\mathrm{nal}^{93}$. Por supuesto, las cuestiones sustantivas deben mantenerse delimitadas; pero se estima que los avances procesales hacia la uniformidad son muy relevantes. Se sugiere, a este respecto, que se pueden encontrar apoyos deductivos en la literatura del Derecho Penal. Es imposible no estar impresionado por el hecho de que el material jurídico en temas penales (ciertamente en todo lo que concierne al proceso, procedimiento y pena) que se conserva en los libros del Digesto 47-49 es todo de los últimos clásicos - Scaevola, Calistrato, Venuleio, Papiniano, Paulo, Ulpiano, Macer, Marciano, Hergemoniano, Modestino, etc. - y gran parte proviene de los trabajos «de officio ${ }^{94}$. ¿ Se puede no suponer que es, al menos en parte, una consecuencia de la difusión general del procedimiento magistral? El procedimiento magistral trataba las materias civiles y penales con detenimiento. Paralelamente, la antigua, clara y obvia separación de lo civil y penal estaba llegando a ser algo confusa respecto al procedimiento: Tener en cuenta al Derecho Penal y distinguir entre lo civil y

${ }_{88}$ Con el consecuente debilitamiento de la precisión terminológica advertida antes, p. 420 y n. 22.

${ }^{89}$ Ej., Buckland, Textbook (Segunda edición), ParTsch, Longi Temporis Praescriptio, 105 ss.

${ }_{90}$ Cf. Jones, Studies in Roman Government and Law, 143 ss. (= 31 JRS, 36 ss).

91 Cf. C. 3.3.2; C. 2.57.1; BuCKLAND, cit. 663 ss.

92 Cf. Pugliese, Guida allo Studio della Civiltá romana antica, I. 447 ss., ArangioRuiz, Storia, ch. 10, Jolowicz, Historical Introduction (Segunda edición) 411 ss., LEVY, 45 BIDR.

93 Ej. VOLTERRA, BIDR cit.

94 Por lo que se refiere a esto, ver más recientemente, Dell'Oro, I Libri 'de Officio' nella Giurisprudenza Romana (1960). 
lo penal se convirtió en una necesidad que no había sido sentida previamente. En resumen, paradójicamente, es la creciente similitud del proceso la que hubiese hecho más notable la separación entre ellos en contenido e interés. En el campo de la prescripción, a pesar de toda la legislación sobre demandas civiles en el periodo post-clásico, no se dice ni una palabra sobre el ámbito penal ${ }^{95}$. Nuevamente existe una pequeña duda de si el tardío periodo clásico, y, especialmente, el principio del tercer siglo, fue un periodo crucial en el desarrollo del Derecho Penal en general, con, por ejemplo, el surgimiento de la distinción de «honestiores» y "humiliores» y la difusión general del «ius gladii» ${ }^{96}$.

Pero, por recapitular: la generalización de un periodo de 20 años en materias fiscales por Severo y Caracalla ya había sido observada. Una vez más es una constitución de los mismos emperadores la que primero concedió el periodo de 20 años en la «longi temporis praescriptio» ${ }^{97}$, como era, "uno ictu». Si se acepta, como desearía, el carácter genuino de la referencia a los 20 años en la C. 9.22.12., la forma ciertamente podría sugerir algo que está bien establecido. En vista de la conexión del interés del Imperio entre lo fiscal, lo provincial y el Derecho Penal, ¿podría haber sido una gran ampliación si - quizás por decreto del periodo de Severo y/o Caracalla, o quizás simplemente por la actividad de Papiniano, Paulo o Ulpiano en su capacidad magistral - el plazo fiscal de 20 años hubiese sido también extendido al delito? La "longi temporis praescriptio», por supuesto, afecta particularmente a las relaciones entre individuos, y la preservación del decreto de Severino con ello, imponiendo esta limitación para los litigios de los ciudadanos particulares «inter se", es, consecuentemente, comprensible. Pero el campo del delito, no obstante, afecta más directamente al Estado y, por ello, si la prescripción penal fuese una extensión de los logros fiscales, no debería sorprendernos que esté menos documentado que el origen del que deriva.

Por tanto, podría aventurar la posibilidad de un origen en Severino para la prescripción penal de veinte años, referida en la C. 9.22.12; y, si eso fuera así, podría ser más comprensible el silencio de los últimos emperadores sobre la cuestión de la limitación temporal penal —en

95 Cf. Volterra (BIDR cit., 71 ss.). Ver también AmElotTi, op. cit., 165 ss. Se piensa que los factores considerados más arriba sugieren la irrelevancia de la analogía de Volterra de obligatio (op. cit., 65 ss.) y su insistencia en la separación de la pretensión penal y civil. (pp. 69-70).

96 Cf. Jones, Studies, cit., 51 ss (esp. 63 ss.) (= Studies Robinson II, 918 ss.); CARDASCIA, 28 RHDFE (1950) 305 ss., 461 ss.

97 C. 7.33.1. 
contraste con el aumento de la legislación en cuestiones civiles-. Después de todo, desde el punto de vista aquí avanzado, el periodo de limitación podría haber existido hacía cerca de un siglo, ya en la época de Constantino.

III

Esto nos lleva a las disposiciones legislativas que se creen que están en contradicción con la C. 9.22.12. Hasta aquí han sido interesantes las prescripciones especiales o los ejemplos imprescriptibles, por supuesto, pero, como se ha dicho, no originan ninguna dificul$\operatorname{tad}^{98}$.

Primero, debemos considerar la C. 9.22.17:

C. 9.22 .17 (Impp. Diocletianus et Maximianus AA. et CC. Menelao) «Sicuti falso testamenti vel codicillorum scriptura temporis intervallo firmari non potest, ita vera quae iure subsistit non evanescit. (1) Si itaque de fide delicti vel per accusationem vel per privatum iudicium quereris, rector provinciae tunc demum eos exhiberi de quibus interrogatio fieri potest iubebit, si prius ceteris indiciis fuerit commotus ». (A. D. 294).

De esto, el Profesor Volterra afirma ${ }^{99}$ (si se acepta mi traducción),

"Está claro que Diocleciano, hablando de la "accusatio falsi», inmediatamente después del principio de imprescriptibilidad afirmado en el "principium", parece considerar esta acusación también como sujeta al mismo principio y no suponer la existencia de un plazo de veinte años ».

Sugiero, con todo el respeto, que la deducción es errónea. La realidad, en mi opinión, es simplemente que el Emperador dice, de forma razonable, que un buen escrito no se invalida por el paso del tiempo más que uno falso se valida en similares circunstancias, y el 17.1 debe leerse exclusivamente sobre esta base - sin ninguna alusión a la criminalidad perpetua- - Un buen documento sigue siendo bue-

98 Ver antes, p. 418 y nn. 9-16. De un modo parecido ninguna dificultad es causada por el lenguaje de 48.5 .30 (29).9 (Ulpiano, lib.iv de adult.):

"Eum autem, qui per vim stuprum intulit vel mari vel feminae, sine praefinitione huius temporis accusari posse dubium non est, cum eum publicam vim committere nulla dubitatio est $"$.

Esto forma parte simplemente de la prescripción de 5 años para el "adulterium», diciendo que no es aplicable a la violación, estupro (Ver posteriormente, sin embargo, C.Th. 9.24.3).

99 BIDR cit., 61. 
no y uno malo, malo; pero eso no determina los posibles procedimientos respecto a, o basados en cualquiera de ellos.

Más importante, de hecho el más importante de los textos supuestamente en conflicto, es la C.Th. 9.19.2.

C.Th. 9.12.2 (Imp. Constantinus A. ad Maximum P(refectum) $\mathrm{U}(\mathrm{rbi}))$ " Cum in praeterito is mos in iudiciis servaretur, ut prolatis instrumentis, si ea falsa quis diceret, a sententia iudex civilis controversiae temperaret eoque contingeret, ut imminens accusatio nullis clausis temporibus petitorem possessoremve deluderet, commodum duximus ut, etsi alteruter litigantium falsi strepitum intulisset, petitori tamen possessorive momentum prolatorum instrumentorum conferret auctoritas, ut tunc civili iurgio terminato secunda falso actio subderetur. (1) Volumus itaque, ut primum cesset inscriptio. Sed ubi falsi examen inciderit, tunc ad morem pristinum quaestione civili per sententiam terminata acerrima fiat indago argumentis testibus scripturarum conlatione aliisque vestigiis veritatis. Nec accusatori tantum quaestio incumbat nec probationis ei tota necessitas indicatur, sed inter utramque personam sit iudex medius nec ulla quae sentiat interlocutione divulget, sed tamquam ad imitationem relationis, quae solum audiendi mandat officium, praebeat notionem, postrema sententia quid sibi liqueat proditurus. (2) Ultimum autem finem strepitus criminalis, quem litigantem disceptantemque fas non sit excedere, anni spatio limitamus, cuiuis exordium testatae apud iudicem competentem actionis nascetur auspicium : capitali post probationem supplicio, si id exigat magnitudo commissi, vel deportatione ei qui falsum commiserit imminente ». (A.D. 326).

El Profesor Volterra ${ }^{100}$, desde el principio, toma el «nullis...temporibus» como si indicase una ilimitada posibilidad de persecución después de Diocleciano - y, de este modo, como si demostrase el anacronismo de los 20 años en la C. 9.2.12. Al respecto, me atrevería a sugerir un significado completamente diferente para la constitución de Constantino. Propongo que la frase en cuestión se refiere a la duración de los procedimientos, una vez comenzados, y no al periodo en el que la persecución podía ser iniciada.

Después de las «Leges Iuliae iudiciorum publicorum et preivatorum» ${ }^{101}$, una vez comenzada una acción civil, tenía que ser finalizada, a lo sumo, dentro de dieciocho meses ${ }^{102}$; pero no existe indicio de algo similar para los procedimientos penales. A pesar de que hemos tenido noticia de otras disposiciones de esta legislación en asuntos pe-

100 BIDR cit., p. 60; ver asimismo AMELotTI, cit., 161.

101 Cf. Rotond, Leges Publicae Populi Romani, 448.

102 Cf. G. IV. 104. 
nales - algunas de ellas bastante detalladas ${ }^{103}$ _, no hay ninguna mención a un límite en el procedimiento penal. No es probable que pudiera existir este silencio si la legislación hubiese tratado este punto. Una vez más, es significativo que el primer precepto general sobre la duración de los procedimientos penales que encontramos haga referencia a un año de duración - viene después de Constantino y bien puede haber sido inspirado por la disposición de la C.Th. 9.19.2.2. Las constituciones a las que se refiere son la C.Th. 9.36.1. y 2 del 385 y 409 d.C, respectivamente ${ }^{104}$. Justiniano, por supuesto, señaló un periodo de dos años y alteró las disposiciones anteriores de acuerdo con esto ${ }^{105}$.

No hay indicación de limitación alguna sobre la duración de los procedimientos en la literatura jurídica, con la excepción de una especial disposición respecto al "peculatus» ${ }^{106}$. La deducción clara es la ausencia de cualquier limitación en la duración de los procedimientos en el tercer siglo y después ${ }^{107}$.

Ante esto propongo que, en la C.Th. 9.19 .2 pr., Constantino se ocupó de la posible no limitación de la duración de una "accusatio" en relación con la acción civil, donde surgía la cuestión de "falsum». Y yo mantengo que la confirmación de esto se encuentra en la disposición del 2.2, que indica que en lo sucesivo las cuestiones penales deberán solucionarse en el plazo de un año ${ }^{108}$ - la disposición que he sugerido fue generalizada por la C.Th. 9.36.1 y 2. Por lo tanto, la disposición de Constantino no tiene nada que ver con la prescripción del delito como tal, y no es obstáculo para la aceptación de la C. 9.22.12, que indica una prescripción de 20 años, ya aceptada con carácter general en el momento de su pronunciamiento.

\section{IV}

Si la C. 9.22.12 es correcta respecto al «sicut...», queda otro punto por tomar en consideración: ¿Cuál es la importancia del decreto?

103 E.g. D. 48.2.3.

104 Ver también Lex Rom. Burgund. 31.5.

105 Cf. p. 421 y n. 24 ante.

106 48.13.11.6. Las referencias a un año en $48.16 .15 .5,50.1 .25 .1$ y 50.4 .7 . todas tienen que ver con el comienzo de los procedimientos después de la acusación, no con su terminación una vez comenzados.

107 Ver también en sentido similar Mommsem, cit., 488, n. 1; AmelotTI, cit., 170, n. 181

${ }^{108}$ Nada se encuentra en su Edictum de Accusatinibus (ver, e.g. F.I.R.A. I, 458 ss). 
La observación de Amelotti apuntada anteriormente continúa siendo válida $^{109}$ y podemos tratar de discernir cómo Diocleciano vino a hacer el pronunciamiento. Yo propongo la siguiente base:

Sabemos que "falsum» surgió de la «lex Cornelia nummaria testamentaria» de Sulla ${ }^{110}$. La amplia definición encontrada en P. Sent. V. $25.3^{111}$ — "Falsum est, quidquid in veritate non est, sed pro vero adseveratur»- se alcanza a través de la recepción de otros supuestos dentro de las penas de la "lex» por "senatusconsulta» ${ }^{112}$ y por edictos imperiales y constituciones ${ }^{113}$. El grupo de casos abarcados era naturalmente enorme ${ }^{114}$. No es sorprendente, por lo tanto, que hubiera variaciones en algunos aspectos entre algunas de esas manifestaciones del "crimen falsi». Por poner algún ejemplo, existe la diferencia entre los miembros de una familia y los «extranei», que se incluyen por escrito a sí mismos en legados dentro del testamento de alguien ${ }^{115}$; una vez más, la aparente permanencia de responsabilidad para la "suppositio partus" y el limitado grupo de quienes podían perseguirlo ${ }^{116}$. Algunos delitos, como la «suppositio partus» y la falsificación de moneda (que de hecho con el tiempo llegó a ser «maiestas» $)^{117}$, se consideraban obviamente más atroces que otras formas de "falsum». Y la confirmación puede encontrarse en que, a pesar de que las penas de la propia «lex» fueron originariamente «aquae et igni interdictio", posteriormente "deportatio" y "publicatio bonorum» o, para los esclavos, el "ultimum supplicium»" ${ }^{118}$, existen textos que expresamente nos narran casos que eran tratados con más indulgencia que lo que la «lex» ordenaba ${ }^{119}$. Existe un amplio grupo de formas de "falsum» y variaciones en los procesos y en las penas. Quizás nuestra constitución C.9.22.12 no ha reparado en formular que, a pesar de tales variaciones, las acciones de "falsum» sólo prescribían ${ }^{120}$ por el pla-

\footnotetext{
109 Ver ante., p. 421 y n. 23.

110 Cf. Rotondi, cit., 356.

111 Ver también Coll. 8.6.

112 E.g., Coll. 8.7, D. 48.10 .6 pr., 1.7, 22 (passium), 15.3.

113 E.g., 47.11.6.1; 48.10.15 pr., 15.1, 32.1.

114 Ver los principales títulos, D. 48.10, C. 9.22, P.Sent. V. 25, Coll. 8.6 y 7.

11548.10 .1 .8 .

$11648.10 .19 .1 ; 48.10 .30 .1$ y cf. 48.2 .11 .1 .
}

117 Sobre eso, ver, C.9.24.2.1. y cf. C.Th. 9.38.6 y C 1.4.3.4; para castigos más severos por falsificación, cf. 48.10.8; ver en general GRIERSON, "Roman Law of Counterfeiting" en Essays in Roman Coinage presented to Harold Mattingly (1956), pp. 240 y ss.

118 Para el desarrollo, cf. 48.10.33; 48.10.1.13. y P. Sent. V. 25.7-13.

119 Cf. 47.11.6.2; 48.10.32.1; 48.10.13.1; 48.10.21.

120 Para n.b., accusatio suppositi partus; y cf. p. 418 ante y n. 15. 
zo de veinte años ${ }^{121}$. Los supuestos que debían ser tratados, por condena, con penas más leves son, sin embargo, susceptibles de persecución tanto como los casos más graves. Esto, por supuesto, se reconoce como conjetura.

$* * *$

En resumen, sostengo que el punto de vista tradicional es muy elogiable, es decir, que en el tercer siglo había surgido, en ausencia de otra disposición específica, una limitación de la responsabilidad penal de veinte años.

121 El delito apuntado en 29.5.13 no es una forma de falsum, pero otra disposición del senadoconsulto que implicaba una forma de falsum fue apuntada en Coll. 8.7.1. 